\title{
In Vitro Analysis of the Fracture Resistance of Cad-Cam Cerasmart Molar Crowns with Different Occlusal Thickness
}

\author{
Roberto Sorrentino*1, Yuko Nagasawa ${ }^{2}$, Arianna Infelise ${ }^{3}$, Giovanni Bonadeo ${ }^{3}$ and Marco Ferrari ${ }^{3}$ \\ ${ }^{1}$ Department of Neurosciences, Reproductive and Odontostomatological Sciences, University Federico II of Naples, Italy \\ ${ }^{2}$ Department of Restorative and Biomaterials Sciences, School of Dentistry, Meikai University, Japan
}

${ }^{3}$ Department of Medical Biotechnologies, University of Siena, Italy

Received: March 05, 2018; Published: March 15, 2018

*Corresponding author: Roberto Sorrentino, Department of Neurosciences, Reproductive and Odontostomatological Sciences, School of Dental Medicine, University Federico II of Naples, Naples, Italy, Email: errestino@libero.it

\section{Abstract}

Purpose: To compare the fracture resistance and mode of failure of CAD-CAM monolithic hybrid composite-nanoceramic crowns with different occlusal thickness.

Materials and Methods: Thirty CAD-CAM monolithic hybrid composite-nanoceramic crowns with different occlusal thickness were randomly distributed into 3 groups: $0.5 \mathrm{~mm}, 1.0 \mathrm{~mm}$ and $1.5 \mathrm{~mm}$. The restorations were cemented onto human molars with a self-adhesive resin cement. The specimens were loaded until fracture; the fracture resistance and mode of failure were recorded.

Results: The strength of the specimens progressively increased with occlusal thickness; the highest resistance to fracture was reported in $1.5 \mathrm{~mm}$-thick crowns but no statistically significant differences were noticed among the experimental groups ( $>0.05)$. The ultrathin crowns $(0.5 \mathrm{~mm})$ were mainly interested by unrestorable fractures.

Conclusion: The occlusal thickness of CAD-CAM monolithic crowns did not influence the fracture resistance of restorations but it significantly affected their mode of failure; the occlusal thickness of such restorations can be reduced up to a lower bound of $1.0 \mathrm{~mm}$ in order to keep sufficient strength to with stand occlusal loads; CAD-CAM monolithic hybrid composite-nanoceramic crowns showed sufficient fracture resistance to be used in molar regions but not in an ultrathin configuration $(0.5 \mathrm{~mm})$.

\section{Introduction}

In the last decade, new production technologies and implemented material science has led to a massive introduction of metal-free restorations in clinical dental practice [1-3]. Moreover, innovative digital workflows allowed a variety of Computer Aided Design-Computer Aided Manufacturing (CAD-CAM) block materials to gain popularity among dentists; such machinable materials are available as composite, resin-infiltrated ceramic and ceramic blocks, to be used prevalently as single tooth restorations $[4,5]$. The use of monolithic metal-free crowns presented both mechanical and biological advantages: the absence of veneering porcelain eliminates the risk of ceramic chipping and the prosthetic room reduction due to the absence of metal framework and opaque layer allows for tooth tissues preservation. Moreover, metal-free restorations offer a more natural tooth-like appearance, resulting in astounding esthetics and optimal translucency [6,7]. On the basis of minimal intervention dentistry, the preservation of tooth structures is crucial and produced a significant change in preparation designs that have become more and more conservative particularly in posterior regions, taking advantage of adhesive cementation techniques $[2,8,9]$. Nonetheless, besides manufacturers' guidelines, there is no consensus about a minimum recommended thickness for metal-free posterior restorations $[1,10,11]$. In this scenario, machinable CAD-CAM composite blocks have gained popularity because of undeniable advantages, such as reliable mechanical properties, high esthetic potential and wear behavior similar to opposing dental enamel [5]. Digital chairside techniques as well as production procedures allow for standardized processing of restorative materials, reduce fabrication times and improve costeffectiveness [6,12]. Ceramic blocks may suffer from their intrinsic brittle nature and could abrade opposite dentition whereas 
composite blocks may be negatively affected by material wear and could show reduced fracture resistance $[12,13]$.

To overcome these drawbacks, mmanufacturers developedinnovative formulations for chair side CAD-CAM materials, combining the advantages of ceramics, just like color stability and durability, with the positive properties of composite resins, such as reduced abrasiveness and improved flexural strength [14,15]. Although fracture patterns were similar, significant differences between CAD-CAM composite and ceramic crowns were reported; indeed, resin-based restorations exhibited longer endurance limits before unrestorable fractures [16]. Furthermore, it was pointed out that CAD-CAM composite-based blocks have better shock absorbing properties than similar ceramic materials, although these results still remain partly controversial $[17,18]$. To date, very few studies are available about Cerasmart and its advantages and limitations should be investigated and better understood both in vitro and in vivo [14]. The present in vitro study aimed at comparing the fracture resistance and mode of failure of CAD-CAM monolithic hybrid composite-nanoceramic single crowns (SCs) with different occlusal thickness cemented onto human molars. The null hypotheses stated that there was no association between the occlusal thickness and either the fracture resistance

a. and the mode of failure

b. of CAD-CAM monolithic hybrid composite-nanoceramic SCs.

\section{Materials and Methods}

\section{Specimen Preparation}

As reported in a previous investigation [1]. 30 extracted human maxillary third molars were used for the study. Teeth with caries and/or previous restorations were excluded; only sound teeth with similar $( \pm 1 \mathrm{~mm})$ bucco-lingual, mesio-distal and corono-apical dimensions were included in the study. Dental plaque, calculus and external debris were removed with an ultrasonic scaler. In order to simulate the oral environment, the teeth were stored in an incubator at $37^{\circ} \mathrm{C}$ at $90 \%$ relative humidity until the execution of the mechanical tests.
Each tooth was embedded in a block of self-curing acrylic resin (Caulk Orthodontic Resin, Dentsply caulk, Milford, DE, USA) surrounded by a stainless steel cylinder with the long axis perpendicular to the base of the block, leaving $1 \mathrm{~mm}$ of the root exposed. In order to dissipate the heat generated during the polymerization of the resin, the specimens were continuously moistened with water spray. A thin layer of polyvinylsiloxane impression material (Flexitime, Heraeus Kulzer, Hanau, Germany) was applied on dental roots to simulate the periodontal ligament. Each tooth was covered with a powder for digital scanning (Cerec Optispray, Sirona Dental, Salzburg, Austria) and three-dimensionally (3D) scanned by means of a laboratory optical digital scanner (GC Aadva Lab Scan, GC, Tokyo, Japan). The 3D shape of each tooth was digitized, so as to use it for the fabrication of CAD-CAM monolithic crowns (Figure 1). Standardized tooth preparations were performed with high-speed diamond rotary cutting burs under constant water cooling, according to the following geometry: $1 \mathrm{~mm}$ axial reduction, 0.7 peripheral rounded minichamfer shoulder placed $0.5 \mathrm{~mm}$ above the cemento-enamel junction, $12^{\circ}$ of total occlusal convergence; all preparation angles were rounded. The 30 specimens were randomly divided into 3 groups of 10 specimens each and different occlusal thickness preparations were performed as follows: $0.5 \mathrm{~mm}$ (group 1), $1.0 \mathrm{~mm}$ (group 2) and $1.5 \mathrm{~mm}$ (group 3).

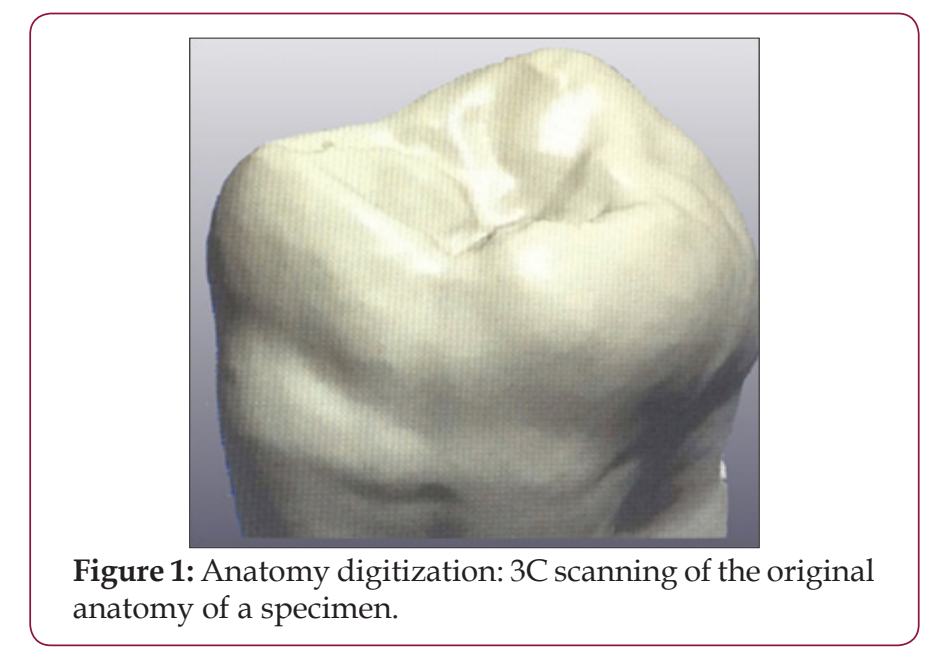

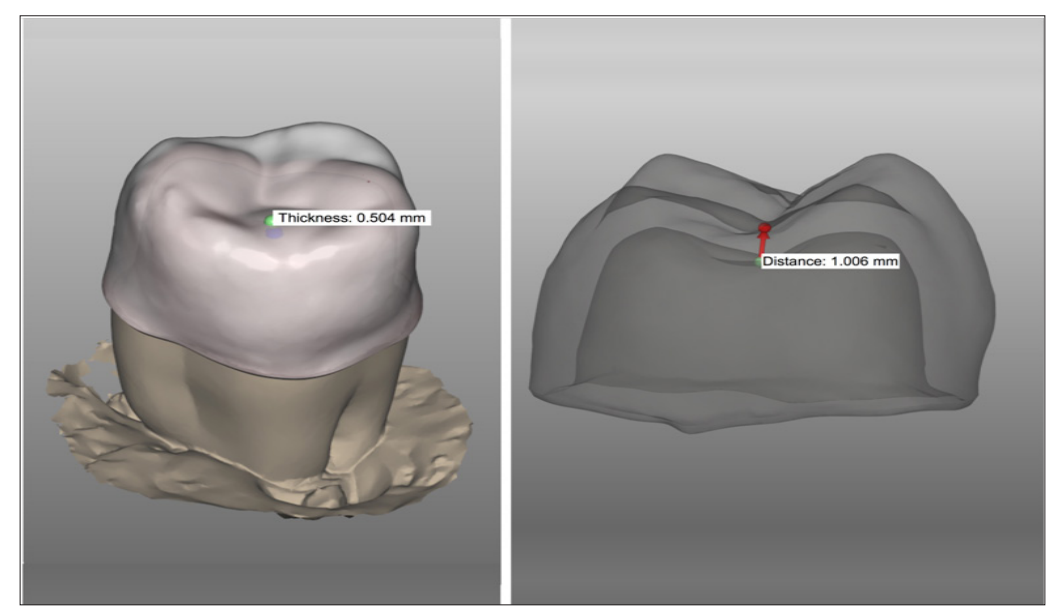

Figure 2: CAD process: occlusal thickness and distance measurements. 
As previously described, each abutment tooth was scanned and digitized and 30 monolithic hybrid composite-nanoceramic SCs were designed by means of a dedicated CAD software (Exocad DentalCAD, Exocad GmbH, Darmstadt, Germany). The restorations of group 1, 2 and 3 presented with an occlusal thickness of 0.5 , 1.0 and $1.5 \mathrm{~mm}$ respectively (Figure 2). The monolithic hybrid composite-nanoceramic crowns were designed according to the original shape of each specimen (Figure 3). Cement spaces of 70 and $50 \mu \mathrm{m}$ were simulated at level of the intaglio surface and of the minichamfer shoulder respectively. After the CAD-CAM restorations were fabricated (Cerasmart blocks, GC), the internal surface of

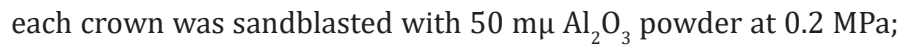
then, they were put in an ultrasonic cleaner with ethanol for $60 \mathrm{~s}$ and cleaned with steam for $60 \mathrm{~s}$. finally, the inner surfaces of the restorations were silanized (Ceramic Primer II, GC) and dried. Dualcure self-adhesive universal resin cement (G-Cem LinkAce, GC, Tokyo, Japan) was used to lute the restorations. The crowns were seated onto the abutment teeth with finger pressure and then $5 \mathrm{~kg}$ were applied onto each crown for 5 min by means of a dedicated cementation appliance. Cement excess was removed with a micro brush and each surface was light-cured for $40 \mathrm{~s}$ with a LED curing unit (Elipar S10, 3M ESPE, Seefeld, Germany). A layer of glycerin gel was applied on the margin of each crown to block oxygen inhibition and polymerization was completed for $40 \mathrm{~s}$ on each surface.

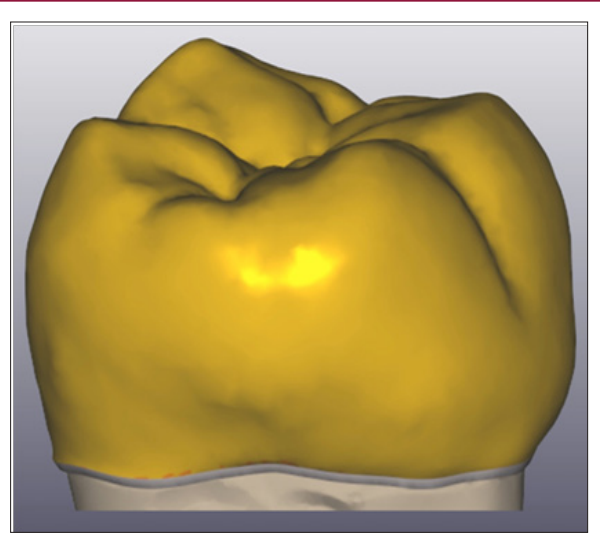

Figure 3: CAD finalization: monolithic lithium disilicate single crown designed in accordance with the original digitized anatomy.

\section{Load to Fracture Test}

A universal loading machine (Triaxial Tester T400 Digital, Controls srl, Cernusco, Italy) was used to statically load the samples. Load to fracture was performed using a $1.0 \mathrm{~mm}$ stainless steel hemispherical tip placed in the occlusal fossa. The experimental load was applied at a crosshead speed of $1 \mathrm{~mm} / \mathrm{min}$ in a direction parallel to the longitudinal axis of the tooth (Figure 4). All the specimens were loaded until fracture and the maximum breaking loads were recorded in Newtons $(\mathrm{N})$ by a computer (Digimax Plus, Controls srl) connected to the loading machine. The failure mode was visually evaluated using a stereomicroscope at $10 \mathrm{x}$ magnification (Zeiss OpMi1, Zeiss, Oberkochen, Germany) and, in case of fracture, the fracture pattern was examined using a scanning electron microscope (SEM Jeol, Tokyo, Japan).

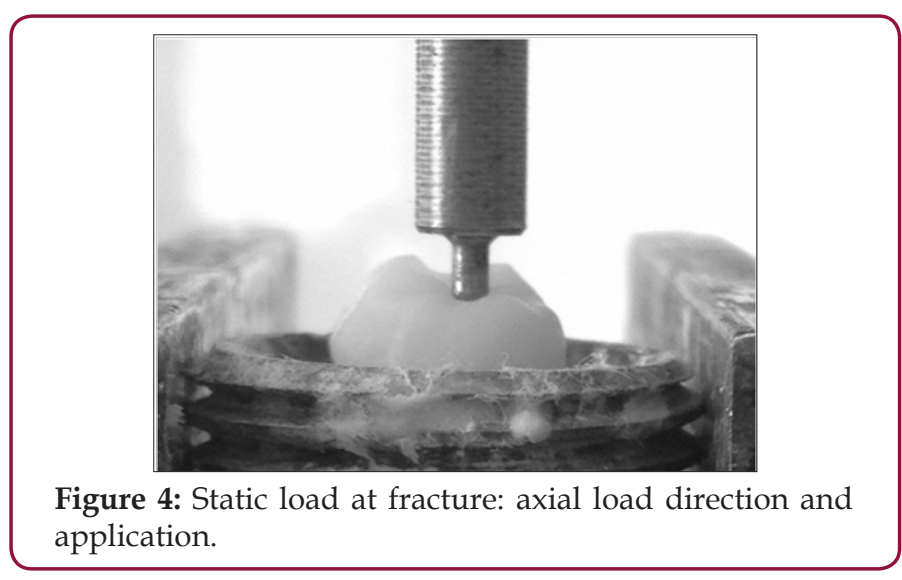

\section{Statistical Analysis}

The recorded data were statistically analyzed with dedicated software (SPSS 13.0, SPSS Inc., Chicago, IL, USA). The KolmogorovSmirnov test was used to verify the normality of data distribution. The fracture values were analyzed with the one-way ANOVA; in order to verify whether statistically significant differences were found among the experimental groups, the Tukey's post hoc test with Bonferroni's correction was applied. In all the analyses the level of significance was set at $\alpha=0.05$.

\section{Results}

In the present study, the survival rates of molar CAD-CAM monolithic hybrid composite-nanoceramic SCs were 10\%, 60\% and $100 \%$ in the experimental groups 1, 2 and 3 respectively. The fracture resistance values progressively increased with occlusal thickness (Table 1). The unrestorable fractures showed catastrophic adhesive failures of the SCs exposing either the cement layer and/ or the dental surface (Figures $5 \& 6$ ). conversely, the restorable fractures caused cohesive microcracks of the hybrid compositenanoceramic cores in the occlusal region, particularly at level of the load application area (Figure 7). The ultrathin crowns $(0.5 \mathrm{~mm})$ were mainly interested by unrestorable fractures whilst thicker crowns were predominantly affected by restorable fractures. No fractures of supporting dental abutments were noticed. Comparable fracture strength values were noticed between the study groups ( $p>0.05$ ) while statistically significant differences were pointed out in the mode of failurebetween group 2 and the other experimental groups (Table 2).

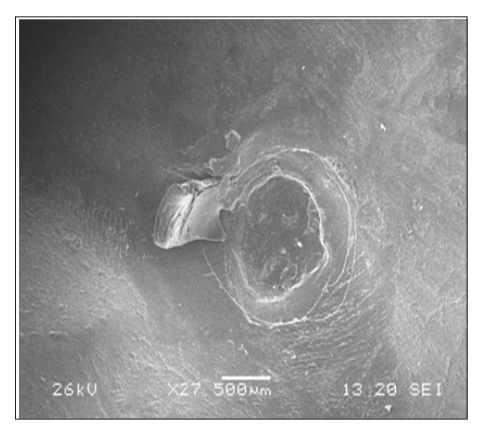

Figure 5: Hybrid composite-nanoceramic crown chipping: SEM image of a cohesive microcrack of the Cerasmart core in the occlusal region at level of the load application area. 


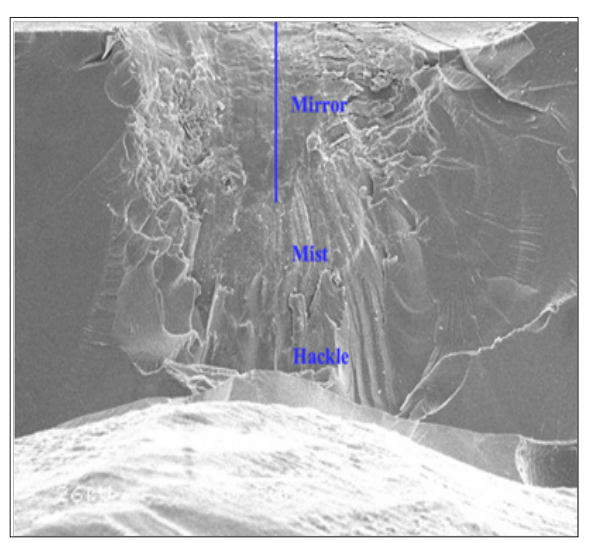

Figure 6: Crack propagation: SEM detail of an unrestorable fracture with the typical mirror (smooth) to mist (slightly rougher) to hackle (very rough) features originating from the load application occlusal area.

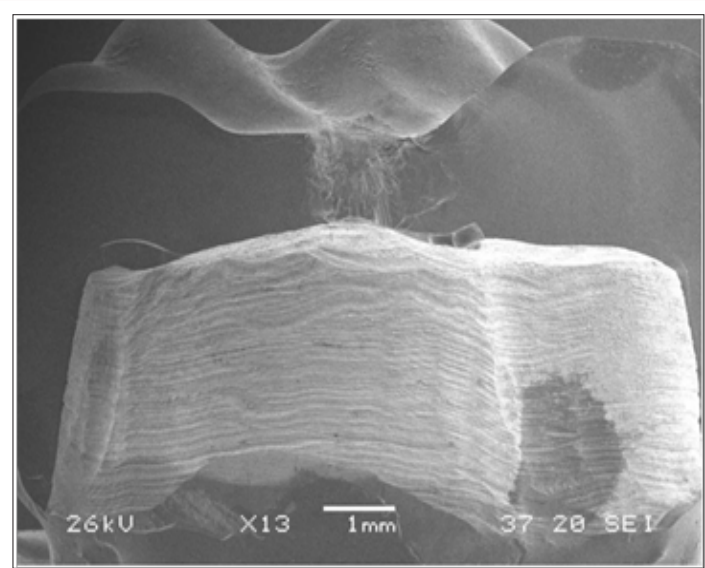

Figure 7:Hybrid composite-nanoceramic crown failure: cross-sectional SEM image of a complete fracture of the Cerasmart core starting from the load application area.

Table 1: Load at fracture (in Newtons) and failure patterns (R: restorable, U: unrestorable) of the experimental specimens.

\begin{tabular}{|c|c|c|c|c|c|c|}
\hline \multirow{2}{*}{$\mathbf{n}$} & \multicolumn{2}{|c|}{ Group $1(0.5 \mathrm{~mm})$} & \multicolumn{2}{|c|}{ Group $2(1.0 \mathrm{~mm})$} & \multicolumn{2}{|c|}{ Group 3 (1.5 mm) } \\
\hline & Fracture load (N) & Failure mode & Fracture load (N) & Failure mode & Fracture load (N) & Failure mode \\
\hline 1 & 416.18 & $\mathrm{U}$ & 1015.28 & $\mathrm{R}$ & 1139.20 & $\mathrm{R}$ \\
\hline 2 & 898.48 & $\mathrm{U}$ & 314.77 & $\mathrm{U}$ & 460.56 & $\mathrm{R}$ \\
\hline 3 & 916.90 & $\mathrm{U}$ & 1379.12 & $\mathrm{U}$ & 1511.18 & $\mathrm{R}$ \\
\hline 4 & 1432.44 & $\mathrm{U}$ & 1449.84 & $\mathrm{U}$ & 1190.09 & $\mathrm{R}$ \\
\hline 5 & 1253.17 & U & 660.47 & U & 925.85 & $\mathrm{R}$ \\
\hline 6 & 835.49 & $\mathrm{U}$ & 1050.34 & $\mathrm{R}$ & 1511.14 & $\mathrm{R}$ \\
\hline 7 & 849.53 & $\mathrm{U}$ & 829.74 & $\mathrm{R}$ & 902.12 & $\mathrm{R}$ \\
\hline 8 & 958.48 & $\mathrm{U}$ & 1208.47 & $\mathrm{R}$ & 1444.26 & $\mathrm{R}$ \\
\hline 9 & 855.40 & $\mathrm{R}$ & 595.01 & $\mathrm{R}$ & 1340.17 & $\mathrm{R}$ \\
\hline 10 & 834.76 & $\mathrm{U}$ & 1505.96 & $\mathrm{R}$ & 686.79 & $\mathrm{R}$ \\
\hline Mean $( \pm \mathrm{SD})$ & $925.08( \pm 269.29)$ & - & $1000.90( \pm 398.06)$ & - & $1111.36( \pm 360.84)$ & - \\
\hline$\%$ & - & R: $10 \%, \mathrm{U}: 90 \%$ & - & R: $60 \%, \mathrm{U}: 40 \%$ & - & R: $100 \%, \mathrm{U}: 0 \%$ \\
\hline
\end{tabular}

Table 2: Results of One-Way ANOVA and Tukey's post hoc test with Bonferroni's correction (level of significance: $a=0.05$ ); same letters indicate $\mathrm{n}$ statistically significant differences.

\begin{tabular}{|c|c|c|}
\hline & Fracture load Sig. & Mode of failure Sig. \\
\hline Group $1(0.5 \mathrm{~mm})$ & A & $\mathrm{a}$ \\
\hline Group $2(1.0 \mathrm{~mm})$ & A & ab \\
\hline Group $3(1.5 \mathrm{~mm})$ & A & b \\
\hline
\end{tabular}

\section{Discussion}

Aaccording to the results of the present investigation, the null hypothesis

a. Was accepted, since there were not statistically significant differences in the fracture resistance of CAD-CAM monolithic Cerasmart SCs; conversely, the null hypothesis.

b. Was rejected, as statistically significant differences were evidenced in the mode of failure between the experimental groups in relation to the occlusal thickness.

From a clinical point of view, the recorded cohesive occlusal microcracks can be considered repairable, since they could be polished intraorally without impairing function. Similarly to previous investigations, the samples were experimentally fractured at a crosshead speed of $1 \mathrm{~mm} / \mathrm{min}$. Cerasmart is a high density nanoparticle-filled composite resin material containing 71\% filler particles by weight [14]. Machinable CAD-CAM polymer-based materials performed better in flexural tests than analogous ceramic materials andthis was due to their relatively high flexural strength combined with a low flexural modulus; consequently, machinable resin-infiltrated materials can withstand occlusal forces by means of an increased elastic deformation before fracture, being more flexible and less brittle than purely ceramic materials $[14,19]$. Cerasmart showed flexural characteristics very similar to that of human dentin and it exhibited a higher mean modulus of resilience than those of other machinable CAD-CAM polymer-based and ceramic blocks; in that, it was reported to be a viable option for single-unit restorations [14,19].

The wall and occlusal thicknesses influence the fracture strength of CAD-CAM composite block restorations [11]. Although several studies are proposing very conservative metal-free restorations with ultrathin (i.e. up to $0.5 \mathrm{~mm}$ ) occlusal thickness, 
scientific data regarding their clinical predictability are lacking, prevalently referring to case reports, mainly in posterior areas [8]. Resin infiltrated ceramic block materials demonstrated adequate wear resistance for load bearing restorations but they require at least similar material thickness as lithium dislocates [20]. Due to the mechanical and biomimetic properties of Cerasmart, it could be speculated that good clinical results can be obtained with reduced available prosthetic space and non ideal preparation design and thickness [14]. As it can be manufactured in very reduced thickness, Cerasmart could be used used in minimally invasive treatment strategies, just like in worn dentition [10]. Since the recorded fracture values exceeded the mean maximum chewing forces in posterior areas (i.e. up to $900 \mathrm{~N}$ ) [21]. All the experimental groups showed the potential to withstand physiological occlusal loads; however, in accordance with the recorded mode of failures, the fracture stability of ultrathinSCs $(0.5 \mathrm{~mm})$ is not recommendable for clinical use in posterior segments.

Similarly to previous investigations, the results of the present analysis suggested the possibility to reduce the occlusal thickness of CAD-CAM Cerasmart crowns up to a safety bound of 1.0 $\mathrm{mm}$, limiting the invasiveness of the preparation and keeping a significant amount of dental tissues [10,11]. It is worth noticing that the resistance to fracture progressively increased with occlusal thickness and the failure mode became more favorable; consequently, differently from the behavior of ceramic materials, thicker cores could enhance the clinical longevity of Cerasmart restorations thanks to the shock adsorbing capability of the high density nanoparticle-filled composite resin matrixx [17]. Furthermore, the clinical performances are strongly related both to the type of resin cement and to the accuracy of adhesive procedures $[6,12,19]$. Several variables could affect the results of static testing, just like specimen storage, die material, cementation technique and crosshead speed and this could explain the heterogeneity of data reported in the literature. Although resistanceto fracture was reported not to be influenced by luting agents [22]. In the present investigation all the specimens were kept hydrated prior to mechanical tests and were luted onto natural molars with a dualcure self-adhesive universal resin cement to simulate a real clinical situation. The formation of an adhesive "monoblock" [23].

Probably contributed to increase the fracture resistance of the SCs, allowing the cement to implementthe elastic stress adsorbing behavior of the nanoparticle-filled composite resin matrix and this could be expected to happen clinically as well, strengthening the restorative system. Although dynamic testing is useful to achieve information about fatigue damages, static axial load tests still represent the first step to assess the fracture resistance of dental materials [24]. However, such analyses would notify about the ultimate strength of materials in order to optimize the geometry of the samples but it is worth noticing that clinical fractures mainly occur because of fatigue. Consequently, the results obtained with static investigations have to be integrated with those obtained from dynamical testing. It is not possible to apply laboratory information directly to clinical recommendations, since the clinical scenario is never completely simulatedby in vitro conditions [25].
As a consequence, the results of the present invitro study have to be validated clinically since only a static vertical load was evaluated.

\section{Conclusion}

Within the limitations of the present in vitro study, the following conclusions can be drawn:

a. the occlusal thickness of CAD-CAM Cerasmart crowns did not influence the fracture resistance but affected the mode of failure of the restorations.

b. the occlusal thickness of CAD-CAM Cerasmart crowns can be reduced up to a lower bound of $1.0 \mathrm{~mm}$ keeping a sufficient strength to withstand occlusal loads.

c. CAD-CAM Cerasmart crowns showed sufficient fracture resistance to be used in molar regions but not in anultrathin configuration $(0.5 \mathrm{~mm})$.

As it is in accordance with the results of previous investigations, the present in vitro study has to be considered a confirmative investigation on the possibility to use CAD-CAM monolithic hybrid composite-nanoceramic crowns in posterior regions.

Further clinical researches will be necessary to validate the results of the present analysis under functional loading.

\section{Acknowledgement}

The authors would like to thank GC Europe (Leuven, Belgium) for the technical support and the fabrication of the CAD-CAM monolithic Cerasmart crowns used in the present investigation.

\section{References}

1. Sorrentino R, Triulzio C, Tricarico MG, Bonadeo G, Gherlone EF, et al. (2006) In vitro analysis of the fracture resistance of CAD-CAM monolithic zirconia molar crowns with different occlusal thickness.J Mech Behav Biomed Mater 61: 328-333.

2. Fuzzi M, Tricarico MG, Ferrari Cagidiaco E, Bonadeo G, Sorrentino R, et al. (2017) Nanoleakage and internal adaptation of zirconia and lithium disilicate single crowns with knife edge preparation. J Oss Perio Prostho 9: $262-274$

3. Joda T, Zarone F, Ferrari M (2017) The complete digital workflow in fixed prosthodontics: a systematic review.BMC Oral Health 17:124.

4. Giordano R (2006) Materials for chairside CAD/CAM-produced restorations. J Am Dent Assoc 137: 14S-21S.

5. Rosentritt M, Hahnel S, Engelhardt F, Behr M (2017) In vitro performance and fracture resistance of CAD/CAM-fabricated implant supported molar crowns. Clin Oral Invest 21: 1213-1219.

6. Zarone F, Ferrari M, Mangano FG, Leone R, Sorrentino R (2016) “Digitally Oriented Materials": Focus on Lithium Disilicate Ceramics. Int J Dent 18: 9840594.

7. Sen N, Us YO (2017) Mechanical and optical properties of monolithic CADCAM restorative materials. J Prosthet Dent 4: S0022-3913(17)3043530443.

8. Fabbri G, Zarone F, Dellificorelli G, Cannistraro G, De Lorenzi M, et al. (2014) Clinical evaluation of 860 anterior and posterior lithium disilicate restorations: retrospective study with a mean follow-up of 3 years and a maximum observational period of 6 years. Int J Periodontics Restorative Dent 34: 165-177.

9. Borelli B, Sorrentino R, Goracci C, Amato M, Zarone F, et al. (2015) Evaluating residual dentin thickness following various mandibular 
anterior tooth preparations for zirconia full-coverage single crowns: an in vitro analysis. Int J Periodontics Restorative Dent 35: 41-47.

10. Mainjot AK, Dupont NM, Oudkerk JC, Dewael TY, Sadoun MJ (2016) From Artisanal to CAD-CAM Blocks: Stateof the Art of Indirect Composites. J Dent Res 95: 487-495.

11. Sieper K, WilleS, Kern M (2017) Fracture strength of lithium disilicate crowns compared to polymer-infiltrated ceramic-network and zirconia reinforced lithium silicate crowns. J Mech Behav Biomed Mater 74: 342 348.

12. Vanoorbeek S, Vandamme K, Lijnen I, Naert I (2010) Computeraided designed/computer-assisted manufactured composite resin versus ceramic single-tooth restorations: a 3 -year clinical study. Int] Prosthodont 23: 223-230.

13. Quinn GD, Giuseppetti AA, Hoffman KH (2014) Chipping fracture resistance of dental CAD/CAM restorative materials: part I-procedures and results. Dent Mater 30: 99-111.

14. AwadaA, Nathanson D (2015) Mechanical properties of resin-ceramic CAD/CAM restorative materials. J Prosthet Dent 114: 587-593.

15. Coldea A, Swain MV, Thiel N (2013) Mechanical properties of polymerinfiltrated- ceramic-network materials. Dent Mater 29: 419-426.

16. Shembish FA, Tong H, Kaizer M, Janal MN, Van Thompson P, et al. (2016) Fatigue resistance of CAD/CAM resin composite molar crowns. Dent Mater 32: 499-509.

17. Menini M, Conserva E, Tealdo T, Bevilacqua M, Pera F, et al. (2013) Shock absorption capacity of restorative materials for dental implant prostheses: an in vitro study. Int J Prosthodont 26: 549-556.

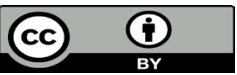

This work is licensed under Creative Commons Attribution 4.0 License

Submission Link: https://biomedres.us/submit-manuscript.php
18. de Kok P, Kleverlaan CJ, de Jager N, Kuijs R, Feilzer AJ (2017) Mechanical performance of implant-supported posterior crowns. J Prosthet Dent 114: 59-66.

19. Goujat A, Abouelleil H, Colon P, Jeannin C, Pradelle N, et al. (2017) Mechanical properties and internal fit of 4 CAD-CAM block materials. J Prosthet Dent 26: pii:S0022-3913(17) 30162-30172.

20. Lawson NC, Bansal R, Burgess JO (2016) Wear strength, modulus and hardness of CAD/CAM restorative materials. Dent Mater 32: 275-283.

21. Varga S, Spalj S, Lapter Varga M, Anic Milosevic S, Mestrovic S (2011) Maximum voluntary molar bite force in subjects with normal occlusion. Eur J Orthod 33: 427-433

22. WeyhrauchM, Igiel C, Scheller H, Weibrich G, Lehmann KM (2016) Fracture strength of monolithic all-ceramic crowns on titanium implant abutments. Int J Oral Maxillofac Implant 31: 304-309.

23. Tay FR, Pashley DH (2007) Monoblocks in root canals: a hypotetical or a tangible goal. J Endod 33: 391-398.

24. Sun T, Zhou S, Lai R, Liu R, Ma S, et al. (2014) Load-bearing capacity and the recommended thickness of dental monolithic zirconia single crowns. J Mech Behav Biomed Mater 35: 93-101.

25. Anusavice KJ, Kaka K, Ferree N (2007) Which mechanical and physical testing methods are relevant for predicting the clinical performance of ceramic-based dental prostheses? Clin Oral Implant Res 18: 218-231.

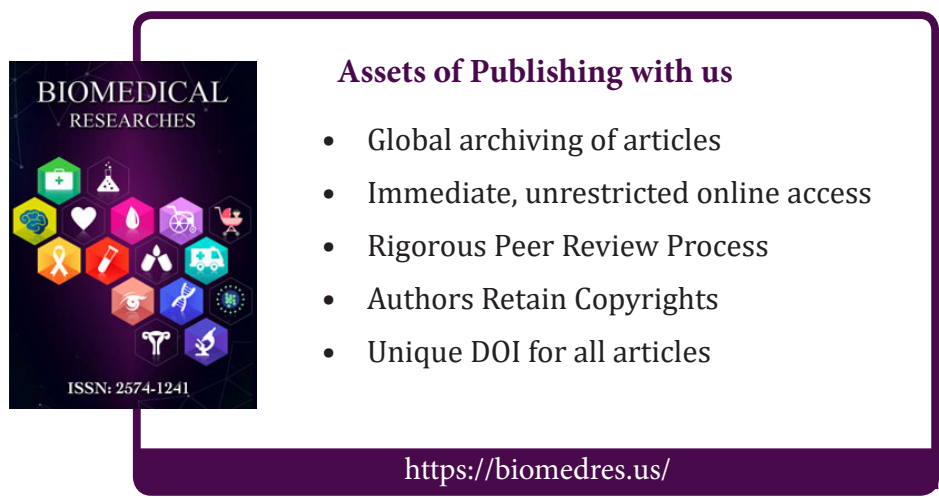

